Tom Rüdebusch

CSCW

Generische Unterstützung von Teamarbeit in verteilten DV-Systemen 


\section{Tom Rüdebusch}

\section{$\operatorname{cscW}$}

Generische Unterstützung von Teamarbeit in verteilien DV-Systemen 
Die Deutsche Bibliothek - CIP-Einheitsaufnohme

Rüdebusch, Tom:

CSCW : generische Unterstützung von Teamarbeit in verteilten

DV-Systemen / Tom Rüdebusch. - Wiesbaden : DUV, Dt.

Univ.-Verl., 1993

(DUV : Informatik)

Zugl.: Karlsruhe, Univ., Diss., 1992

ISBN 978-3-8244-2043-8 ISBN 978-3-322-87883-0 (eBook)

DOI 10.1007/978-3-322-87883-0

Der Deutsche Universitäts-Verlag ist ein Unternehmen der Verlagsgruppe Bertelsmann International.

(C) Deutscher Universitäts-Verlag GmbH, Wiesbaden 1993

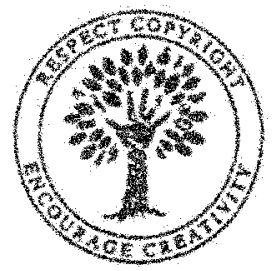

Das Werk einschließlich aller seiner Teile ist urheberrechtlich geschützt. Jede Verwertung außerhalb der engen Grenzen des Urheberrechtsgesetzes ist ohne Zustimmung des Verlags unzulässig und strafbar. Das gilt insbesondere für Vervielfältigungen, Übersetzungen, Mikroverfilmungen und die Einspeicherung und Verarbeitung in elektronischen Systemen. 


\section{Geleitwort}

Viele Arbeiten in den Verwaltungs-, Entwicklungs- und anderen Abteilungen der Großund mittelständischen Unternehmen beruhen auf Arbeitsvorgängen, an denen mehrere Personen beteiligt sind. Die Mittel der Zusammenarbeit wie auch ein koordiniertes Vorgehen beeinflussen hier wesentlich Effektivität und Effizienz und damit die Leistungsfähigkeit der Unternehmen. Weiter ist zu beobachten, daß in zunehmendem Maße an Arbeitsplätzen vernetzte Rechner zur Verfügung stehen. Diese Infrastruktur kann in Verbindung mit neuen Software-Technologien mehreren Personen die Zusammenarbeit durch ein 'Überbrücken' zeitlicher und räumlicher Distanzen sowie durch Hilfsmittel zur Koordination erleichtern. Alle oft sehr unterschiedlichen Ausprägungen des rechnergestützten gemeinsamen Arbeitens werden mit dem Begriff 'Computer-Supported Cooperative Work' (CSCW) zusammengefaßt.

In einer ersten genaueren Betrachtung unterscheidet man grob zwischen dem 'asynchronen' und dem 'synchronen' kooperativen Arbeiten. Das asynchrone kooperative Arbeiten kennzeichnet Arbeitsschritte, die nicht gleichzeitig stattfinden. Ein typisches Beispiel ist die Bearbeitung eines Versicherungsschadens durch mehrere Sachbearbeiter unterschiedlicher Funktion in einer Versicherung. Hier sind verschiedene Rollen und Verantwortlichkeiten als Bestandteile der gesamten Vorgangsbearbeitung festgelegt. Im Gegensatz hierzu finden synchrone kooperative Arbeiten zeitgleich statt. Ein Beispiel ist das gemeinsame zeitgleiche Editieren eines Dokuments. Die rasante Entwicklung im Bereich der Multimedia-Technologie hat hier auch Audio-Video-Konferenzen als festen Bestandteil der Forschung und Entwicklung von CSCW-Systemen etabliert. Die meisten CSCWSysteme basieren entweder auf Text, Text und Graphik oder Audio und Video. Diese Ausrichtungen bedingen unterschiedliche implementierungstechnische Fragestellungen, die in der nächsten Generation von CSCW-Systemen medienübergreifend betrachtet werden sollten.

Aus praktischer Sicht muß man-neben den a-priori für $\mathrm{CSCW}$ ausgelegten Anwendungen-Lösungen anstreben, die auch bestehende Anwendungen in einen CSCWKontext einbinden. Ansätze für gemeinsame Arbeitsbereiche beruhen hier beispielsweise auf der Erweiterung graphischer Fenstersysteme.

Die meisten heutigen CSCW-Systeme sind ausschließlich auf ein Arbeitsgebiet wie 'nur' ein gemeinsames Editieren von Texten oder Bildern, 'nur' eine Vorgangsbehandlung oder 'nur' die Realisierung eines gemeinsamen Fensters ausgelegt. 
In der vorliegenden Arbeit nimmt Herr Rüdebusch zunächst eine umfassende Klassifikation für den Stand der Technik von CSCW vor. Dabei werden als wesentliche Kriterien die Art der Interaktion, der Koordination und der Verteilung angegeben. Bestehende CSCW-Systeme werden nach dieser Klassifikation analysiert und katalogisiert.

Daraus leitet der Autor eine Modellierung von Interaktions- und Koordinationsmustern als Bestandteil einer generischen Anwendungsentwicklungsunterstützung ab. Zentral ist hier die Idee der Erweiterung von Einbenutzer- zu sogenannten Teamobjekten. Interaktion wird dabei als grundlegender Mechanismus zum kooperativen Arbeiten gesehen. So kann beispielsweise zwischen der synchronen und asynchronen Interaktion bezüglich einzelner (Team-)Objekte unterschieden werden. Koordination wird als zur Interaktion orthogonales Konzept modelliert. Hierzu werden u. a. unterschiedliche Team- und Aufgabenstrukturen, Rollen, Teams und Aufgaben als Konstrukte definiert. Diese abstrakte Beschreibung erlaubt, die unterschiedlichsten Ausprägungen von CSCW-Systemen und Anwendungen zu spezifizieren.

Im praktischen Teil der Arbeit wird zur Beschreibung verteilter Teamarbeit eine eigene Sprache definiert und eine konkrete Entwicklungsunterstützung für kooperative Szenarien vorgestellt. Deren Einsatz wird anschließend anhand beispielhafter CSCW-Anwendungen veranschaulicht.

Damit muß das vorliegende Buch von Herrn Rüdebusch als eine sehr präzise strukturierte und kompakte Abhandlung im Gebiet CSCW angesehen werden. Zunächst erlaubt sie dem Leser, sich in kurzer Zeit in dieses oft sehr diffus und unklar wirkende Forschungsgebiet einzuarbeiten. Danach bieten die erarbeitete Modellierung und das darauf aufbauend realisierte System Grou PIE die generelle Möglichkeit, Teamarbeiten zu spezifizieren und somit CSCW-Anwendungen auf einer abstrakteren Ebene zu entwickeln. Durch die hohe Wiederverwendbarkeit müssen die prinzipiellen Probleme nicht für jede Anwendung von neuem gelöst werden. Herr Rüdebusch hat ganz klar und deutlich eine wesentliche Notwendigkeit im Bereich von CSCW aufgedeckt und umgesetzt: weg von der singulären Anwendungsentwicklung hin zu einer Anwendungsgenerierung. 


\section{Inhaltsverzeichnis}

1 Einleitung 1

2 Computer-Supported Cooperative Work (CSCW) 5

2.1 Einführung zentraler Begriffe . . . . . . . . . . . . . . . . 6

2.2 Anwenderaspekte . . . . . . . . . . . . . . . . 11

2.2.1 Beschreibung und Klassifikation kooperativer Systeme . . . . . . 11

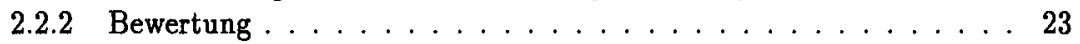

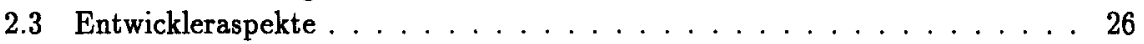

2.3.1 Entwicklungs- und Laufzeitunterstützung für kooperative Anwen-

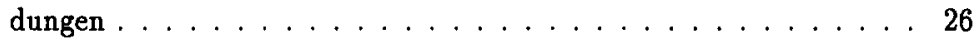

2.3 .2 Bewertung . . . . . . . . . . . . . . . 30

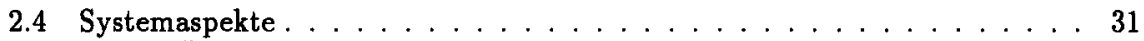

2.4 Übersicht . . . . . . . . . . . . . . . . . . 31

2.4 .2 Benutzerschnittstelle .................. 31

2.4.3 Konkurrenz . . . . . . . . . . . . . . . . . 34

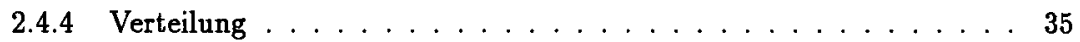

2.4 .5 Kommunikation . . . . . . . . . . . . . 36

2.5 Zusammenfassung und Folgerungen .................. 37

3 Modellierung verteilter Teamarbeit 39

3.1 Anforderungen ........................ 40

3.2 Grundlegende Ideen . . . . . . . . . . . . . . . . . . . . . 42

3.3 Interaktion in verteilter Teamarbeit-ein generischer Ansatz . . . . . . 47

3.3.1 Problematik und Anforderungen . . . . . . . . . . . . 47

3.3 .2 Lösungsideen . . . . . . . . . . . . . . . . . . 48

3.3.3 Interaktionsmodellierung . . . . . . . . . . . . . . 50

3.4 Koordination in verteilter Teamarbeit-ein generisches Rahmenwerk . . . . 59

3.4.1 Problematik und Anforderungen . . . . . . . . . . . . . . 59

3.4 .2 Lösungsideen . . . . . . . . . . . . . . . . . . . 60 60

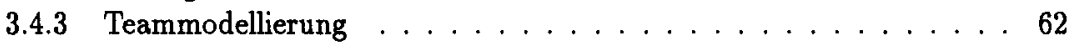

3.4.4 Aufgabenmodellierung .................. 66

3.4.5 Koordinationsmodellierung . . . . . . . . . . . . . 70

3.5 Kooperation als Mittel verteilter Teamarbeit . . . . . . . . . . . . . 72

3.6 Zusammenfassung . . . . . . . . . . . . . . . . . . 74 
4 Unterstützung von Teamarbeit in verteilten DV-Systemen $\quad 77$

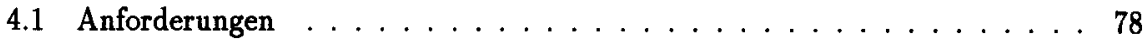

4.2 Lösungsideen . . . . . . . . . . . . . . . . . 79

4.3 Anwendersicht . . . . . . . . . . . . . . . . . 83

4.4 Entwicklersicht . . . . . . . . . . . . . . . . . . 83

4.4.1 Entwicklungs- und Laufzeitunterstützung für kooperative Szenarien 83

4.4.2 Teamobjekte als Bausteine der Interaktionsunterstützung . . . . . . 84

4.4.3 Beschreibungsmittel für die Koordination in Teams und Aufgaben . 92

4.4 .4 Werkzeuge . . . . . . . . . . . . . . . . . . . . 107

4.4.5 Eine kooperative Entwicklungsmethode . . . . . . . . . . 108

4.5 Systemnahe Sicht . . . . . . . . . . . . . . . . . . . 110

4.6 Zusammenfassung . . . . . . . . . . . . . . . . . . . . . . . 112

5 Validierung und Einsatz $\quad \mathbf{1 1 5}$

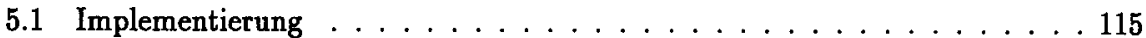

5.2 Einsatz . . . . . . . . . . . . . . . . . . . . . 117

5.2.1 Entwicklung kooperativer Systeme . . . . . . . . . . 117

5.2.2 Weitere Einsatzgebiete . . . . . . . . . . . . . . 125

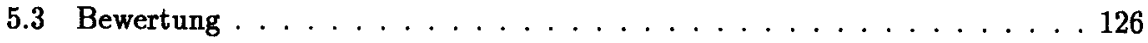

6 Zusammenfassung und Ausblick $\quad 129$

$\begin{array}{ll}\text { Schlußwort } & 133\end{array}$

$\begin{array}{ll}\text { Literaturverzeichnis } & 135\end{array}$

Anhang 159

A Zusammenfassung der Thesen $\ldots \ldots \ldots \ldots \ldots \ldots \ldots$

B Ausgewählte Teamobjektbeschreibungen $\ldots \ldots \ldots \ldots 16 \ldots \ldots$

C Team-, Aufgaben- und Koordinationsbeschreibungssprache . . . . . . 175

C.1 Die Teambeschreibungssprache . . . . . . . . . . . . . . . . . . . . . 176

C.2 Die Aufgabenbeschreibungssprache . . . . . . . . . . . . . . 179

C.3 Die Koordinationsbeschreibungssprache . . . . . . . . . . . . . . . . 185

D Beispiel: Entwicklung kooperativer Szenarien . . . . . . . . . . . . . 191 


\section{Abbildungsverzeichnis}

2.1 Abstraktionsebenen der Basistechnologien kooperativer Anwendungen . . . 31

3.1 Struktur einer Interaktionsbeschreibung . . . . . . . . . . . 58

3.2 Wichtige Beziehungen um Benutzer und Team . . . . . . . . . . 65

3.3 Wichtige Beziehungen um Teamobjekt und Aufgabe . . . . . . . . . . 70

3.4 Wichtige Beziehungen um Team, Aufgabe und Kooperation . . . . . . . . 73

3.5 Grundlegende Elemente verteilter Teamarbeit . . . . . . . . . . . 75

4.1 Konzeptionelle Aufteilung jedes Teamobjekts in ein semantisches und syntaktische Objekte . . . . . . . . . . . . . . . 85

4.2 Aggregationshierarchie eines einfachen kooperativen Graphikeditors . . . 88

4.3 Vererbungshierarchie für Teamobjekte . . . . . . . . . . . 89

4.4 Zusammenhang zwischen und unter Beschreibungen und Instanzen . . . . 9 93

4.5 Eine Entwicklungsmethode für kooperative Szenarien . . . . . . . . . . 109

4.6 Logische Softwarearchitektur der verteilten Kooperationsunterstützung . . 111

5.1 Die komplexe Aufgabe 'Realisiere Kooperation' . . . . . . . . . . . . 118

5.2 Die Teilaufgabe 'informelle Beschreibung' . . . . . . . . . . . . . . . 119

5.3 Interaktion auf dem Entwurfsdokument . . . . . . . . . . . . 120

5.4 Der Interaktionsdemonstrator . . . . . . . . . . . . . . . . 121

5.5 Interaktion auf einer Koordinationsbeschreibung-Sicht A . . . . . . 123

5.6 Interaktion auf einer Koordinationsbeschreibung-Sicht B . . . . . . 124

5.7 Meldungen der Koordinationsauswertung . . . . . . . . . . . 125

5.8 Die Kooperationsunterstützung als 'Enabling Technology' . . . . . . . . . 127 


\section{Tabellenverzeichnis}

2.1 Klassifikation von CSCW-relevanten Systemen nach den unterstützten Distanzen in Raum und Zeit . . . . . . . . . . . . . . . . . . . 8

2.2 Klassifikation von CSCW-relevanten Systemen nach den Kriterien Interaktion, Koordination, Verteilung und Technologie, a) . . . . . . . . . 24

2.3 Klassifikation von CSCW-relevanten Systemen nach den Kriterien Interaktion, Koordination, Verteilung und Technologie, b) . . . . . . . 25

4.1 Beispielhafte Einordnung der auf einigen vordefinierten Teamobjekten möglichen Interaktionen in die vier Interaktionskategorien . . . . . . . . 90 